Original Article

\title{
A RESEARCH CRITIQUE ON THE LIVED-IN EXPERIENCES OF PATIENTS SUBJECTED TO CHEM OTHERAPY IN SELECTED HOSPITALS AT CHENNAI
}

\author{
Malarvizhi $\mathbf{M}^{1} \&$ Bhavani $\mathbf{M}^{2}$ \\ ${ }^{1}$ Lecturer, Faculty of Nursing ${ }^{2}$ Research Scholar, Department of Human Genetics, \\ Sri Ramachandra University, Chennai, Tamilnadu, India. \\ Correspondence : \\ Malarvizhi M
}

Lecturer, Faculty of Nursing, Department of Human Genetics, Sri Ramachandra University, Chennai 600 116, Tamilnadu, India. E-mail : malarvizhi23@gmail.com

\section{Abstract:}

This qualitative study explored the experiences of patients subjected to chemotherapy. The research approach used in this study was phenomenology. Interviews were conducted with fifteen patients subjected to chemotherapy. The study was conducted at selected hospitals at Chennai. From the colaizzi's analysis of the data seven dimensions-eight themes and fifty sub themes were emerged. These findings show that patients subjected to chemotherapy has lot of physical and psychological impacts. There was an association between the socio demographic variables and the lived in experiences of patients subjected to chemotherapy. The association was found between the educational status, type of family and the number of chemotherapy cycle. So, the patients need adequate education on the side effects from the Nurses during each cycle of chemotherapy. Therefore this study is important for nurses working in the cancer unit to make the patient to realize and aware the effectsfaced by the patients subjected to chemotherapy.

Keywords: Phenomenology, lived experiences, Chemotherapy, colaizzi's

\section{Introduction:}

Cancer is a term that is used to refer malignant neoplasms. It is a disease of the cell in which the normal mechanisms of the control of growth and proliferation have been altered. It is invasive, spreading directly to surrounding tissues as well as to new sites in the body. A major concern with the diagnosis and treatment of cancer is the multifocality and multicentrality. The level of distress varies from women to women and within an individual in different situation and role.

Cancer is a disease of cells characterized by a loss of the normal control mechanisms that maintain tissue

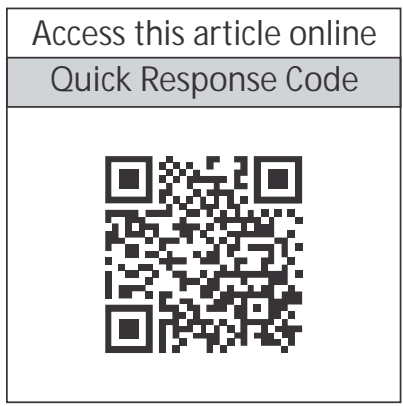

orga $n$ i zation. Chemotherapy is the use of one or more chemical agents to eradicate the cancer cells or to stop tumour progress (Durr and Huff 1994)

Chemotherapy is a systemic intervention and is appropriate when the disease is widespread, the risk of undetectable disease is high, and the tumor cannot be resected and is resistant to radiation therapy. The objective of chemotherapy is to destroy malignant tumor cells without excessive destruction of normal cells. Several types of cancers are now considered curable with chemotherapy, even in advanced stages. The most valuable intervention that can be offered to a patient with cancer is presence of nurse as a caring person. Its dimension consists of verbal expressions of empathy, positive regard, and availability of practical support.

Chemotherapy for malignancies, too advanced for surgery, which accounts for $80 \%$ of all cancers, is a scientific wasteland (Dr. Ulrich Abel, 1990). Amazingly, most patients cope well and face the difficulties in their lives with courage. For many patients the experience brings about a re-appraisal of goals and values, making life richer and more meaningful. Griffin (1998) identified and ranked the symptoms experienced among 155 cancer patients who received chemotherapy. The findings revealed that the 
patients reported on the experience of physical symptom 13 and 7 psychological. Nausea was reported as the most severe symptoms followed by tiredness and loss of hair. The study suggested that a reduction in the severity of some symptoms experienced while receiving chemotherapy and a shift from concerns about physical to psychological issues.

The spiritual and existential matters often marks this phase of illness from diagnosis and treatment because women focus their legacy to their families, children and engaged in planning for their future.(Butler et al 2003)

The emotional side-effects of a person diagnosed as cancer and person who receives chemotherapy are different for everyone. They may even change during the treatment. Before starting chemotherapy, many patients feel anxious about not knowing what to expect and their upcoming treatments. Many patients said that they experienced depression and or emotional stress before and during chemotherapy. Emotional side-effects affects the cancer patient's day-to-day activities. Mitchell (2007) described the social and emotional effects of chemotherapy among 19 patients. The major themes that emerged from the data were, striving for normality, feeling-up, feeling-down, flagging, being sociable and anxiety, about the chemotherapytreatment.

Chemotherapy may bring about another cycle of emotional change, such as anger, denial, guilt, depression and acceptance, as well as impact on other aspects of psychosocial functioning. (Holland and Zitton 1991)

The severity of side-effects varies between individuals, the type of drug administered, and the type of care delivered, including information and other psychosocial interventions. (Coates et al. 1987), Elizabeth L.Mcgarvey(2001) explored the psychological sequel among women with cancer. The findings revealed that they experienced lower self-esteem, poor body image, and lower quality of life, and affects on sexuality.

In Sri Ramachandra Hospital, an average of 15 patients per month are admitted to the inpatient department for chemotherapy treatment in the general wards namely E6 and E7. The investigator felt that it is very important to elicit their lived-in experiences with regard to physical, psychological, social, spiritual, financial and sexual dimensions in order to support and help them to continue with their remaining chemotherapy cycle.

Subjective experiences of individual undergoing chemotherapy allow the practitioner to understand and gain insight on the patient current experience and support him/her with the use of their constructive coping strategies. This helps the nurses to develop strategies for the coping abilities of cancer patient.

So the investigator strongly believes that the feelings which the patients undergo must be explored. This realization, strongly motivated the investigator to do this research critique on lived-in experiences of patients subjected to chemotherapy. Through eliciting the lived-in experiences, it is possible to identify the patients felt needs in all dimensions which in turn will inspire the nursing care.

Nursing is a profession focused on assisting individuals, families and communities in attaining, maintaining, and recovering optimal health and functioning. Modern definitions of nursing define it as a science and an art that focuses on promoting quality of life as defined by persons and families, throughout their life experiences from birth to care at the end of life. So, the nurses play an important role as a supporter, collaborator, and motivator in order to help the patient to have an optimal health and functioning.

\section{Materials and Methods:}

Qualitative study design and phenomenological approach was selected. An in depth interview was conducted. The patients who had been admitted within the study period, and who fulfilled the inclusion criteria were selected with the sample size of 15. Inclusion Criteria were the patients subjected to chemotherapy, Patients who were admitted on the previous day of chemotherapy and staying in the hospital on $1^{\text {st }}$ day of chemotherapy.

Prior to data collection, the necessary permission was obtained from the concerned authorities. Before 
commencing the data collection the investigator got verbal consent from the patients. 15 samples who were admitted for chemotherapy were chosen for the study. The procedure was explained to each patient individually, ensuring comfort and privacy. The study was explained to each participant and informed them that their identity will not be revealed and confidentiality will be maintained and will be utilized for the research purpose only. Informed oral consent was obtained from the patients, and the interviews were audio-taped. An in depth interview was done on a one-to-one basis.

The total time taken for collecting the data from each participant was 30-45 minutes. After the completion of the interview, each patient was given an opportunity to clarify the doubts.

\section{Results:}

Colaizzi's data analysis framework was used to analyze the transcripts in this study. From the analysis of the data, seven dimensions-eight themes and fifty sub themes emerged from the experiences of patients subjected to chemotherapy and they were as follows: The dimensions were physical, psychological, social, economic, spiritual and sexual dimension and various suggestions given by the patients and the level of satisfaction of the Nursing care as expressed by the patients.

\section{Discussion :}

From the analysis of the data, seven dimensions-eight themes and fifty sub themes emerged from the patients subjected to chemotherapy.

\section{A. Physical dimension}

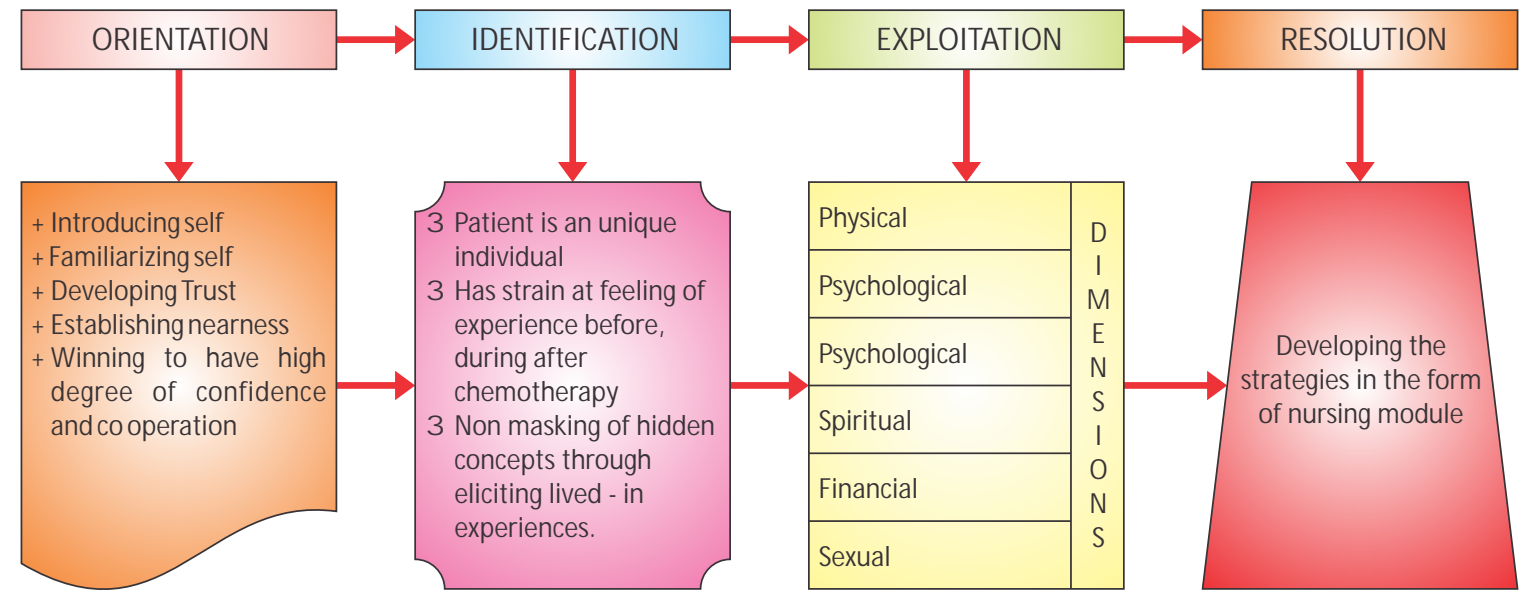

Figure 1 : Conceptual Framework - M odified Peplau's Nurse - Patient Interaction Theory

In physical dimension, the major themes identified were mouth ulcer, anorexia, loss of the sense of taste, hair loss, insomnia, tiredness and unable to perform household activities. All the 15 patients (100\%) have experienced hair loss, mouth ulcer, insomnia and unable to perform household activities, 14 patients (93\%) verbalized that they had anorexia and loss of the sense of taste.

"My hair is falling like a leaf that falls from the tree during winter"

"My mouth ulcer is troubling me like anything because of that I am notable to eat well and to discriminate the taste of different delicious

\section{foods".}

"At night, I am unable to sleep properly because of the generalized weakness".

"I am not able to carry out the household activities like before this makes me stressful".

The above findings were consistent with the study of Warren (1999) who used a qualitative design on reducing the discomfort following chemotherapy. The procedure itself can be associated with severe anxiety and discomfort for the patient. The author reports that the patients' experience of discomfort during and after the procedure can be reduced by proper explanation of the whole 
treatment process before starting the procedure.

\section{B. Psychological dimension}

The identified themes were anger, fear, feeling ashamed, disturbed body image and feeling of happiness. 15 patients $(100 \%)$ felt ashamed of themselves because of their altered body image. 10 patients (67\%) were anxious and worried about their illness and they got irritated often.13 patients(87\%) felt happy about the treatment.

"I get angry and irritated often".

"Everyday I am worried about my children".

"I am much troubled, that there is nobody to whom I can express my grief".

"I havea shameful feeling that my hair is lost".

"I feel that I have lost my self-esteem and body image when l look at others in the society".

"I am so sad because l am ill".

"I am very happy about the chemotherapy treatment".

Marrs (2000) conducted a study by using qualitative techniques of grounded theory in identifying the patients perception of recovery after chemotherapy. 10 men and 15 women were interviewed one month after the first chemotherapy cycle. Verbatim transcript were analyzed for major themes. The results of this study highlights the need for patients concern and support with greater emphasis on their psychosocial needs. Nurses must also consider providing support to patients in the pre admission and recovery phase.

\section{Social dimension}

The identified themes were low self esteem, neighbours thoughts of misperception about the illness, problems with communication.15 patients (100\%) experienced problem in communicating with the neighbours, and had low self esteem. 10 patients(67\%) felt that they had adequate family support.

"I know I can't hide things but some how I will manage this problem and I will take care of myself and my family".

"My friends are helping me to be happy in all the situations".

"I 'm highly emotional and I'm also worried about my prognosis".

The above study findings coincided with the study of Elizabeth (2001) studied how the patients and the significant others felt about their social support services. The findings revealed that higher level of social support received from their peer group than from their family members.

\section{Economical dimension}

The following theme were based on the income. 12 patients(80\%) belong to low socioeconomic background and treatment through military.

"If I hadn't had ECHS scheme, I wouldn't have taken the treatment and I would have died long before".

\section{E. Spiritual dimension}

The identified themes were bargaining and thanking God. 14 patients (93\%) expressed that they would visit the temple and pleaded with God to cure their illness and thanked God for helping them live for a while.

"Of course, I do believe in God, so I pleaded to him to help me to feel better everyday".

"Even my enemy should not get thistype of illness".

"I should thank God for the life given back to me".

"I believe him to make me live for a long time".

The above findings were consistent with the study of Deegan (2007) analyzed the spiritual needs for the hospitalized terminally ill patients. The findings revealed that the spiritual needs should be met by the hospital staff and help the patient to be satisfied in all the other dimensions.

\section{F. Sexual dimension}

The identified theme was satisfaction of the spouse. Six patients (40\%) expressed that they were unable to satisfy their spouse.

"I am not able to satisfy my husband". 


\section{G. Satisfaction Of Nursing Care}

13 patients (87\%) expressed that they were satisfied with the level of care given by the nurses in the ward.

"Nurses are like god and they take care of me well during my illness"

\section{H. Suggestion by The Patients}

13 patients (87\%) suggested to have a separate ward and separate nurse for treating the cancer patients.

\section{"I feel that it would be better to have a separate nurse for each patient, during chemotherapy".}

Four (27\%) patients were illiterate and they were able to recognize the illness early and came for the treatment as soon as they diagnosed to have the illness. Four (27 \%) were graduate and all these patients were diagnosed at the later stage and they have come for their treatment.

12 patients (80\%) were from nuclear family and these patients experienced neglected family and they experienced courageousness to face the illness. Three patients(20\%) were from the joint family and they felt that they were neglected from the family and now in hospital they could not accept the feeling of being alone.

Three (53\%) patients had third cycle of chemotherapy who were able to cope well with their illness and they could face the illness with courage. Five patients (54\%) had more than three cycle but they were not able to face their illness and

\section{References :}

1. Amy, J., Hoffmann., . Relationship among pain, fatigue, Insomnia, and gender in persons with lung cancer. Oncology Nursing Forum. 2007, 34(4), 785-790.

2. Arantzamendi. Psychological needs of patients receiving chemotherapy: An exploration of patient perceptions. European Journal of Cancer Care. 2004. 13(1): 23-31.

3. Arqneis, Ambrissi, P., Beatlie, S., \&Greden,E. Patient's perception of acute pain following therapeutic procedure. Dimens Critical Care Nursing.2000. 9(3): 150-155.

4. Brien, B.J., Rusthoven, J.R. Impact of chemotherapy associated Nausea and vomiting on patients functional status and on costs. Jounal of Supportive Oncology. 2000.149(5), 296-302.

5. David, S., Fischer, Tish Knobt Henry,T. The cancer chemotherapy Handbook. ( $5^{\text {th }}$ ed.). Boston: M osbypublication. 2002.436-442.

6. Deegan, P.E. The lived experience of cancer patient in the recovery process and a shared decision making program to support it. Journal of psychiatric rehabilitation. 2007.31(1), 62-69. they were anxious about their prognosis.

\section{Conclusion:}

Therefore the findings of this study is important for nurses working with patients subjected to chemotherapy. The investigator was able to find out that each patient is unique. Their experience and perception varied from person to person during the process of illness. The essential step in the health care system is to elicit the patient's experiences and to counsel them to strengthens their self esteem by enhancing their compliance and abilities in order to meet their health needs.

Nurses need to expand their time for the patients to meet the other needs than the physical needs. Nurses need to improve their communication, knowledge through staff development programme. Nurses can be the supporter, counselor to the patients and strengthen their self reliance bythe qualitycare.

\section{Acknowledgement:}

Its my pleasure and privelage to record my deep sense of gratitude to Prof.P.V.Ramachandran, Chairman, Nursing Education, College of Nursing, SRU and my guide Dr.Eilean Victoria, Assistant Professor, Sultan Qaboos University, for his inspiring guidance, valuable suggestions, constant encouragement for the completion of the study. I am deeply indebted to my beloved parents. I owe my success to them who made this task possible through their support.

7. Elizabeth, L., M cgarvey.Psychological sequelae and alopecia among women with cancer. Cancer Practice. 2001.9(6), 283-289.

8. Guray, Y.Perception of recovery from patient's point of view. J ournal of Advanced Nursing. 2000.22(9), 241-249

9. Heli vaartio., Kirsi kiviemi. M en's experience and their resources from cancer diagnosis to recovery. Journal of urology. 2003.23(6), 43-46

10. Manoj Pandey.Stress, Anxiety and depression in Cancer patients undergoing chemotherapy. World Journal surgery Oncology. 2006.45(2), 34-38.

11. Polit DF, Beck CT, Nursing Research: Principles and Methods. $7^{\text {th }}$ edition, Philadelphia: Williams and wilkins; 2004.

12. Redeker, N.S.Insomnia, Fatigue, Anxiety, Depression of cancer patients undergoing Chemotherapy. Scholar International Nursing Practice.2000. 14(4), 275-290.

13. Sonja M cilfatrick. Patients experiences of having chemotherapy in a day hospital setting. Journal of Advanced Nursing. 2007.59(2), 264 273 